\title{
Bariatric endoscopy procedure type or follow-up: What predicted success at 1 year in 962 obese patients?
}

\section{다)(우우}

\author{
Authors \\ Bautista ${ }^{1,3}$ \\ Institutions \\ 1 Bariatric Endoscopy Unit, HM Sanchinarro University \\ Hospital, Madrid, Spain. \\ 2 Department of Gastroenterology and Hepatology, \\ Singapore General Hospital, Singapore. \\ 3 Ciber of Obesity and Nutrition Pathophysiology \\ (CIBEROBN), Instituto de Salud Carlos III, Madrid, Spain
}

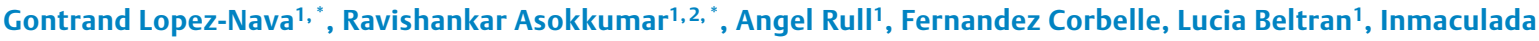

submitted 31.5.2019

accepted after revision 16.8 .2019

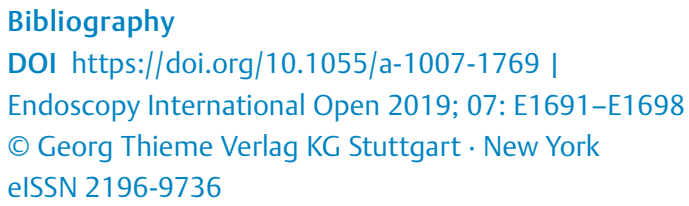

Corresponding author

Ravishankar Asokkumar, Singapore General Hospital Department of Gastroenterology and Hepatology, The Academia 20 College Road Singapore 169608, Singapore Fax: +62273623

ravishnkr03@gmail.com

\section{ABSTRACT}

Background and study aims It is uncertain if the difference in weight loss outcomes between different endoscopic bariatric therapies (EBTs) is technique-related or multidisciplinary team (MDT) follow-up-related. We hypothesized that at 1 year, the weight loss is determined more by adherence to MDT follow-up than by procedure type. We aimed to compare 1 year weight loss outcomes of four different EBTs at a single center with a standardized MDT follow-up.

Patients and methods We prospectively collected and retrospectively analyzed outcomes in 962 patients (female-691, 71.2\%; mean age, $44.8 \pm 10.6$ years, mean BMI, $37.8 \pm 5.9 \mathrm{Kg} / \mathrm{m}^{2}$ ) treated with Intragastric balloons (IGBs) or endoscopic gastroplasty (EG) at HM Sanchinarro University Hospital between March 2012 to January 2017. The procedures were performed by the same endoscopist and followed up by the same MDT. We compared the percentage total body weight loss (\%TWBL) at 1 year. We performed linear and logistic regression to identify predictive factors for weight loss and follow-up adherence at 1 year. Results Four hundred and eighty-one IGBs (Orbera-80.9\%; ReShape Duo-19.1\%), and 481 EG (Apollo ESG-51.3\%; Primary obesity surgery endoluminal-POSE-48.6\%) were performed. Only 480 patients (IGB- 45\%; EG- 55\%) completed 1 year follow-up. Among them, Apollo ESG achieved significantly higher TBWL $(19.5 \pm 13 \%, P=0.035)$, \%TBWL (17.4 \pm $10.2 \%, P=0.025)$, and $\geq 20 \% \operatorname{TBWL}(36.7 \%, P=0.032)$. However, in linear regression after adjusting for variables, only higher initial $\mathrm{BMI}(\mathrm{B}=0.31, P<0.001)$ and higher percentage follow-up attendance $(B=0.24, P<0.001)$ significantly predicted \%TBWL at 1 year in the completion group but not the procedure type $(B=0.02, P=0.72)$. In logistic regression, we observed female sex $(P=0.01)$, high initial BMI $(P<0.001)$, endoscopic gastroplasty $(P=0.04)$, and high 1 -month \%TBWL $(P<0.001)$ significantly predicted followup completion at 1 year.

Conclusions Weight loss at 1 year is dependent on MDT follow-up rather than procedure type. Endoscopic gastroplasty promoted follow-up adherence more than IGBs.

\section{Introduction}

Endoscopic bariatric therapies (EBTs) are evolving to be an effective minimally invasive treatment option for patients with obesity and associated comorbidities [1,2]. EBT is performed either as a primary stand-alone therapy or as a bridge treat-

\footnotetext{
* Drs. Lopez-Nava and Asokkumar: These authors contributed equally.
}

ment before surgery [3]. Currently, three US Food and Drug Administration-approved devices are available for endoscopic treatment of obesity, and a few more are under development [4]. Among them, intragastric balloons (IGBs) and endoscopic gastroplasty (EG) techniques have gained wide popularity and acceptance.

Several studies have established the technical feasibility, reproducibility, safety, and efficacy of IGBs and EG [5-8] 
- Table1 Summary of data published on each technique.

\begin{tabular}{|l|l|l|l|l|c|}
\hline Procedure type (ref) & Publication (n) & Patients $(\mathbf{n})$ & \%TBWL-12 months (95\% CI) & SAE (\%) & SAE needing surgery \\
\hline Orbera [13, 14] & 8 & 8506 & $10 \%(6.6-13.6 \%)$ & $1.5 \%$ & $0.1 \%$ \\
\hline ReShape Duo [15] & 1 & 202 & $14.7 \%(8.02-21.4)$ & $0.03 \%$ & $16 \%$ \\
\hline POSE [20] & 3 & 402 & $10.98(3.48-18.48)$ & $0.03 \%$ & $0.09 \%$ \\
\hline ESG [24] & 9 & 1542 & $16.09 \%(14.24-17.94)$ & $1 \%$ & $21 \%$ \\
\hline
\end{tabular}

( $\triangleright$ Table 1). However, considerable variation exists in shortterm and long-term total body weight loss outcomes $[9,10]$. At 12-month follow-up, Apollo Overstitch sutured gastroplasty (Overstitch; Apollo Endosurgery, Austin, Texas, United States), was shown to achieve total body weight loss (17.6\%) with the effect sustained up to 24 months (20.9\%) [11]. On the contrary, the total body weight loss with IGBs ranged between $11 \%$ and $15 \%$ at 6 months with a risk of weight regain after balloon removal [12-15]. Multiple factors, in addition to the anatomical and neuroendocrine signaling changes, may have influenced the difference in weight loss outcomes [16].

In our prior experience treating 25 patients (body mass in$\operatorname{dex}[\mathrm{BMI}]>30 \mathrm{~kg} / \mathrm{m}^{2}$ ) with Apollo ESG, we found that the number of contacts with the nutritionist and psychologist during follow-up significantly predicted the weight loss at 1 year [17]. Currently, it is unclear if the weight loss results between different EBTs is related to the technique or multidisciplinary team (MDT) follow-up or both. Most centers differ in the nutrition and follow-up protocol and there is a lack of evidence comparing weight loss outcome of different EBTs within an identical follow-up program.

Since 2011, our bariatric endoscopy unit has standardized EBT techniques, adopted a structured post-procedure followup protocol, and performed a variety of EBTs. In this study, we aimed to compare weight loss outcomes of different EBTs within the same follow-up program and evaluated factors that predicted weight loss and follow-up adherence at 1 year.

\section{Patients and methods}

\section{Study design}

We prospectively collected and retrospectively studied data from patients who underwent EBTs at the Bariatric Endoscopy Unit of HM Sanchinarro University Hospital, Madrid, between March 2012 and January 2017. The institutional review board approved the study. All authors had access to the study data and reviewed and approved the final manuscript. All the procedures were performed following the ethical principles detailed in the Declaration of Helsinki and were consistent with the Good Clinical Practices recommendation.

\section{Study patients}

We included 1013 patients who received EBTs during this period. All the patients were referred to our unit after failed conservative therapy at other centers. The EBTs were offered as a selfpay procedure and not covered by health insurance. We discus- sed the different endoscopic bariatric treatment options with the patient and explained the risks, benefits, and cost (EG $>$ IGB) of each procedure. Choice of EBT was decided based on patient preference. We did not have a personalized or preferred treatment approach based on patient profile. All the patients included paid the treatment expenses upfront, and the cost of MDT follow-up was identical. We extracted data on demographics, weight parameters, type of EBT, and compliance to follow-up from our obesity database for analysis. All patients consented to the procedure. We excluded those who were referred for EG after failed IGB.

\section{Intervention}

Procedure

The EBTs performed during this study included Intragastric balIoon placement (Orbera, Apollo Endosurgery, Austin, TX; ReShape Duo, Apollo Endosurgery, Austin, TX) and endoscopic gastroplasty using Apollo Overstitch or Primary obesity surgery endoluminal (POSE, USGI Medical, San Clemente, USA) ( $\triangleright$ Fig. 1). All the procedures were performed by the same endoscopist and were followed-up by the same multidisciplinary team (nutritionist and psychologist). We have previously described in detail our technique on different IGB placement, Apollo ESG, and POSE in our prior publications [7, 8, 18-20].
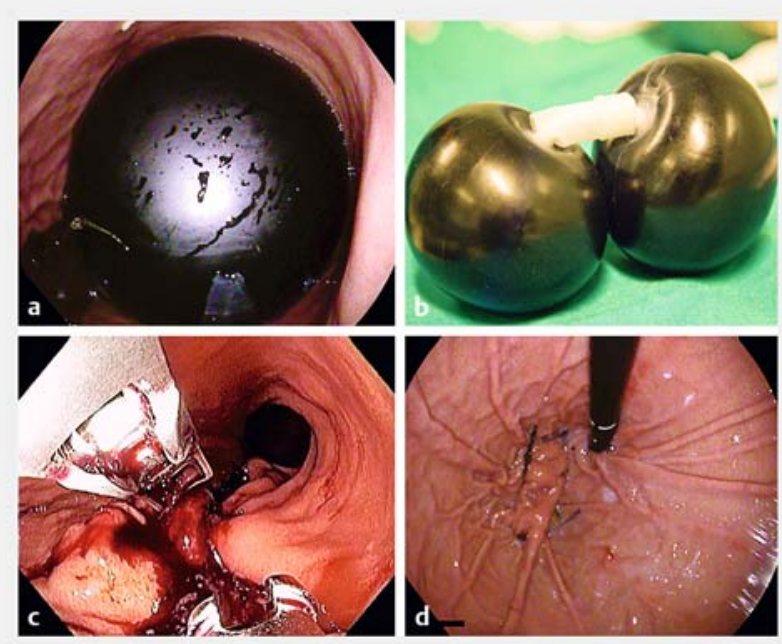

- Fig. 1 Endoscopic image of 4 different EBTs. a Orbera IGB. b ReShape Duo IGB. c Apollo endoscopic sleeve gastroplasty. d POSE. 
We do not routinely perform repeat endoscopy to assess the integrity of the sutures with the endoscopic gastroplasty procedures.

\section{Follow-Up Schedule}

Patients were followed-up weekly or biweekly post-procedure by a nutritionist, psychologist, and physiotherapists. When their condition stabilized, we extended the visits to once a month. We recommend achieving 24 clinic visits over 1 year irrespective of the procedure type. The follow-up program comprised dietary instructions, psychological support, physical activity, and a planned counseling schedule, as well as a timeline for future visits.

Body requirements and individual taste preferences were taken into account while designing the hypocaloric diets. Energy requirements were calculated from the Harris-Benedict formula. Based on the type of physical activity, we decreased caloric intake by about $2.6 \mathrm{MJ} /$ day to induce an approximate weight loss of between 0.5 and $1 \mathrm{~kg} /$ week. In the first month after EG, we maintained patients on a strict liquid diet (4 weeks). We subsequently escalated intake to semi-solid and solid food as tolerated. In the IGB patients, we initiated a solid meal within 2 weeks. We used a Mediterranean type diet in which distributions of principal components were as recommended by the Spanish Society of Community Nutrition [21].

We devised an individualized exercise plan and avoided an increase in intra-abdominal pressure during the first month. We encouraged walking in the initial phase and then progressively increased exercise intensity. There were no other differences in the follow-up plan between the two groups, and the patients were counseled to adhere to follow-up even after balloon explantation.

\section{Study outcomes}

The primary outcome was to compare the weight loss results of four different EBTs at 1 year. Our secondary outcome was to identify factors that predict weight loss and follow-up completion at 1 year.

\section{Study variable description}

We collected information on the following variables.

1. Gender

2. Age: Continuous variable and we classified them into two groups (Group $1 \geq 45$ years; Group $2<45$ years)

3. Type of EBTs: We segregated them into IGB group (Orbera and ReShape Duo IGB), EG group (Apollo ESG, and POSE)

4. Weight Parameters: We measured weight, height, and BMI at baseline and during each follow-up visit. We classified them into two groups based on severity of obesity (Group $1 \geq 40 \mathrm{~kg} / \mathrm{m}^{2}$, and Group $2<40 \mathrm{~kg} / \mathrm{m}^{2}$ ).

5. Attendance to follow-up: We defined it as the percentage of scheduled visits to which the patient went during the first year of follow-up. We divided follow-up attendance in the completion group into three tertiles to understand its relationship with the weight parameters [17].

6. End of Treatment Response: We classified them into two groups. Completion group are those who had weight results recorded at 1 year. Drop-out group are those who failed to complete the 1 year recommended follow-up. Patients were considered drop-out if they had no weight loss results recorded at 1 year irrespective of the prior follow-up attendances.

7. Weight loss parameters: We measured \%TBWL and \%excess weight loss (\%EWL) with the four EBTs at the end of 1 year. We grouped them into those who achieved $\geq 10 \%$ TBWL and those who met $\geq 20 \%$ TBWL.

\section{Statistical analysis}

We expressed continuous variables as mean \pm standard deviation (SD) and categorical variables as a percentage. Comparisons of means between groups were calculated using the Mann-Whitney or Kruskal-Wallis test (for not normally distributed variables). Bivariate analyses of proportionality of distribution of categorical variables were estimated using the Chisquare test. We performed a linear regression analysis to evaluate the relationship of age, gender, initial BMI, procedure type, and $\%$ follow-up attendance on \%TBWL at 1 year. We performed a logistic regression analysis to study the association between age, sex, initial BMI, and procedure type on follow-up adherence at 1 year. We used Kaplan-Meier survival curves to illustrate patient attrition over treatment time. $P<0.05$ was considered statistically significant. We analyzed the data analyses using Statistical Package for the Social Sciences 19.0 software (SPSS Inc., Chicago, Illinois, United States).

\section{Results}

\section{Patient characteristics}

We identified 1013 patients who underwent EBTs during the study period. We excluded 51 cases as they were treated using an alternative swallowable IGB (Elipse, Allurion Technology, United States) device with a shorter follow-up duration (4 months). Among the remaining 962 patients, we treated half ( $50 \%, n=481$ ) using IGBs and the rest with EG. We used Orbera balloon in 389 (40.4\%) and ReShape Duo IGB in 92 (9.6\%) cases, and performed Apollo ESG in 247 (24.3\%) and POSE in $234(25.7 \%)$ patients, respectively ( $\triangleright$ Table 2$)$. The mean number of follow-up visits to the MDT was $7.6 \pm 5.3$ (median-7; IQR7 ; range- 0 to 36$)$. Only half the patients ( $n=480,49.9 \%)$ had weight loss results recorded at 1 year ( $\triangleright$ Fig. 2 ). The remaining patients dropped out from follow-up at a different time interval. Characteristics of the study participants are detailed in - Table 3. Mean age was $44.8 \pm 10.6$ years, and mean BMI was $37.8 \pm 5.9 \mathrm{Kg} / \mathrm{m}^{2}$. The majority of participants were female $(71.2 \%, n=691)$.

\section{Weight loss results with four EBTs}

Among the 480 patients who reached 1 year follow-up (completion group), $45 \%$ were treated with IGBs and $55 \%$ using EG techniques. Overall mean TBWL, \%TBWL and \%EWL of the bariatric program involving the four EBTs were $18 \pm 11.9 \mathrm{~kg}$, $16.3 \pm 9.3 \%$, and $51 \pm 30.8 \%$, respectively. Ninety-one percent achieved $\geq 5 \%$ TBWL $(n=436), 77 \%$ obtained $\geq 10 \%$ TBWL $(n=$ $370)$, and $31 \%$ reached $\geq 20 \%$ TBWL $(n=150)$. Among the EBTs, 
- Table 2 Baseline characteristics of EBTs.

\begin{tabular}{|l|l|l|l|l|l|}
\hline Variables & Orbera & Reshape Duo & Apollo & POSE & P value \\
\hline Age & $42.9 \pm 11$ & $42.3 \pm 12.8$ & $45.9 \pm 9.6$ & $47 \pm 10.1$ & $<0.001$ \\
\hline Sex & $70.2 \%$ & $79.3 \%$ & $70.4 \%$ & $73.1 \%$ & 0.32 \\
\hline Initial BMI & $37.6 \pm 6.7$ & $38.4 \pm 5.2$ & $38.3 \pm 5.7$ & $37.6 \pm 4.8$ & 0.052 \\
\hline BMI $>40 \mathrm{~kg} / \mathrm{m}^{2}$ & $29.6 \%$ & $33.7 \%$ & $36.4 \%$ & $27.8 \%$ & 0.16 \\
\hline EBT, endoscopic bariatric therapy; BMI, body mass index & & & \\
\hline
\end{tabular}

\section{Screened patients $(n=1013)$}

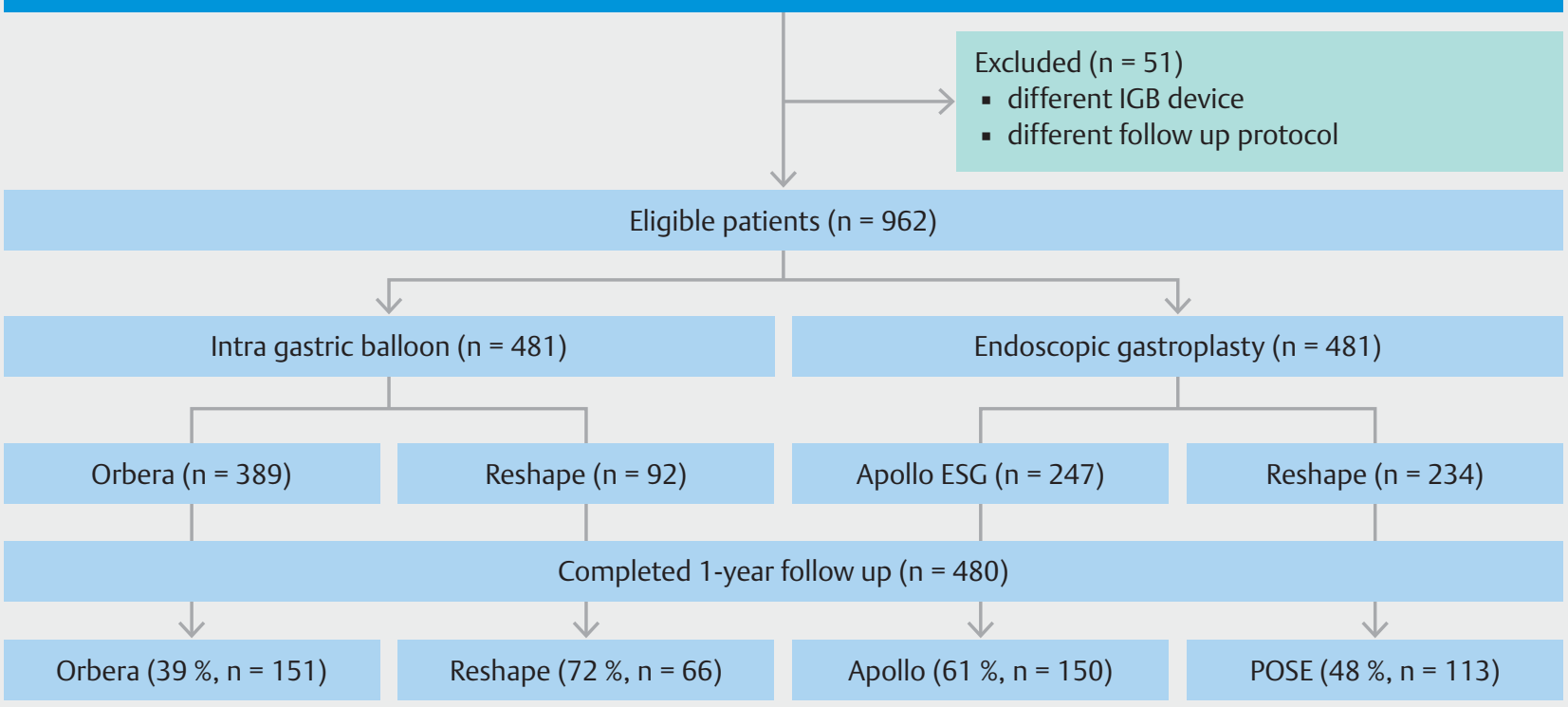

- Fig. 2 Flowchart describing the study population.

- Table 3 Characteristics of study groups.

\begin{tabular}{|c|c|c|c|}
\hline Characteristics & Completion Group $(n=480)$ & Drop-out Group $(n=482)$ & $P$ value \\
\hline Age $\pm S D$, years & $45.3 \pm 11.3$ & $44.3 \pm 10$ & 0.04 \\
\hline - <45 years & $45 \%$ & $55 \%$ & \\
\hline - >45 years & $52 \%$ & $48 \%$ & \\
\hline Gender & & & 0.01 \\
\hline - Male & $44 \%$ & $56 \%$ & \\
\hline - Female & $52 \%$ & $48 \%$ & \\
\hline Initial $\mathrm{BMI} \pm \mathrm{SD}, \mathrm{kg} / \mathrm{m}^{2}$ & $38.5 \pm 5.8$ & $37.2 \pm 6$ & $<0.001$ \\
\hline - $<40 \mathrm{~kg} / \mathrm{m}^{2}$ & $46 \%$ & $54 \%$ & \\
\hline - $\geq 40 \mathrm{~kg} / \mathrm{m}^{2}$ & $59 \%$ & $41 \%$ & \\
\hline Procedure type & & & 0.002 \\
\hline - IGB & $217(45 \%)$ & $264(54 \%)$ & \\
\hline - EG & $263(55 \%)$ & $218(45 \%)$ & \\
\hline
\end{tabular}


- Table 4 Weight loss outcomes with different techniques at 1 year.

\begin{tabular}{|c|c|c|c|c|c|}
\hline Outcomes & $\begin{array}{l}\text { Orbera } \\
(n=151)\end{array}$ & $\begin{array}{l}\text { Reshape Duo } \\
(n=66)\end{array}$ & $\begin{array}{l}\text { POSE } \\
(n=113)\end{array}$ & $\begin{array}{l}\text { Apollo } \\
(n=150)\end{array}$ & $P$ value \\
\hline Mean \pm SD TBWL & $18.7 \pm 12.6$ & $15.3 \pm 8$ & $16.6 \pm 10.6$ & $19.5 \pm 13$ & 0.035 \\
\hline Mean \pm SD \%TBWL & $16.9 \pm 9.3$ & $14.4 \pm 6.7$ & $15.3 \pm 8.7$ & $17.4 \pm 10.2$ & 0.025 \\
\hline$\geq 10 \%$ TBWL & $79.5 \%$ & $74.2 \%$ & $71.7 \%$ & $80 \%$ & 0.337 \\
\hline$\geq 20 \%$ TBWL & $35.1 \%$ & $19.7 \%$ & $25.7 \%$ & $36.7 \%$ & 0.032 \\
\hline Mean \pm SD \%Attendance & $56.3 \pm 26.9$ & $31.9 \pm 23.8$ & $25.1 \pm 17.7$ & $59.3 \pm 25.6$ & 0.001 \\
\hline
\end{tabular}

- Table 5 Weight loss outcomes with different techniques at 6 months.

\begin{tabular}{|l|l|l|l|l|}
\hline Outcomes & $\begin{array}{l}\text { Orbera } \\
\text { (n=228) }\end{array}$ & $\begin{array}{l}\text { Reshape Duo } \\
(\mathbf{n = 5 3 )}\end{array}$ & $\begin{array}{l}\text { POSE } \\
(\mathbf{n = 1 4 8 )}\end{array}$ & $\begin{array}{l}\text { Apollo } \\
(\mathbf{n = 1 9 5 )}\end{array}$ \\
\hline Mean \pm SD TBWL & $17.2 \pm 9.8$ & $13.5 \pm 6$ & $15.6 \pm 8.3$ & $17.7 \pm 9.2$ \\
\hline Mean \pm SD \%TBWL & $15.7 \pm 7.5$ & $12.6 \pm 4.9$ & $14.5 \pm 6.8$ & $16.1 \pm 7.4$ \\
\hline$>10 \%$ TBWL & $82.2 \%$ & $75.6 \%$ & $71.6 \%$ & $83.1 \%$ \\
\hline$\geq 20 \%$ TBWL & $37.2 \%$ & $24.4 \%$ & $24.2 \%$ & $37.7 \%$ \\
\hline Mean \pm SD \%Attendance & $45.3 \pm 26.9$ & $37.4 \pm 23.5$ & $18.5 \pm 15.9$ & $49.6 \pm 27.5$ \\
\hline TBWL, total body weight loss & & & & 0.008 \\
\hline
\end{tabular}

Apollo ESG demonstrated significantly higher mean \pm SD TBWL $(19.5 \pm 13 \mathrm{~kg}, P=0.035)$, \% TBWL $(17.4 \pm 10.2, P=0.025), \geq 20 \%$ TBWL (36.7\%, $P=0.032$ ) and had the highest percentage follow-up attendance at 1 year $(59.3 \pm 25.6 \%, P=0.001)(\triangleright \mathrm{Ta}-$ ble 4). To better compare and synchronize with balloon removal, we analyzed results in patients who completed follow-up at 6 months ( $n=624)$ and found Apollo ESG achieved significantly higher mean \pm SD TBWL $(17.7 \pm 9.2 \mathrm{~kg}, P=0.008)$, \% TBWL (16.1 $\pm 17.4, P=0.007)$, and higher percentage follow-up attendance $(49.6 \pm 27.5 \%, P=0.001)$ ( $\triangleright$ Table 5$)$

We analyzed the relationship between weight loss parameters (TBWL, \%TBWL, \%EWL) and MDT follow-up. We classified attendance to MDT follow-up into three groups: low-attendance ( $T 1$, attendance $<28 \%$ ), medium attendance ( $T 2$, attendance $28 \%-57 \%$ ), and high attendance (T3, attendance $>57 \%$ ) [17]. We found the magnitude of weight loss increased significantly in patients who achieved high MDT follow-up attendance in the completion group at 6 months and 1 year. $(P<0.01)$

( $\triangleright$ Fig. 3 and $>$ Fig. 4 ).

\section{Predictive factors for weight loss and follow-up completion at 1 year}

We performed a linear regression analysis, after controlling for study variables, to identify predictive factors for \%TBWL in the completion group $(n=480)$. We found \%follow-up attendance $(B=0.24, P<0.001)$ and initial $\mathrm{BMI}(\mathrm{B}=0.31, P<0.001)$ predicted higher \%TBWL at 1 year. Type of EBT did not influence weight loss at 1 year $(B=0.02, P=0.72)$. These findings were identical when we performed the analysis at 6 months $(n=$
624). Procedure type did not influence weight loss $(B=0.01$, $P=0.72$ ) ( $\triangleright$ Fig. 5 and $\triangleright$ Fig. 6).

We performed a logistic regression analysis to identify factors that predicted follow-up completion at 1 year in the entire cohort. We found gender (females adhered better), initial BMI (higher BMI-higher adherence), procedure type (endoscopic gastroplasty-longer follow-up), and \%weight loss at 1 month (higher weight loss- longer follow-up) significantly predicted follow-up completion at 1 year ( $>$ Table 6 ). However, when analyzed at 6 months, type of procedure did not predict follow-up adherence $(P=0.2)$.

We performed additional analysis to identify variables associated with high attendance to MDT (T3, attendance $>57 \%$ ), independent of treatment completion at 1 year. We found initial BMI ( $B=0.103, P=0.012)$, female sex $(B=0.12, P=0.002)$ and $\%$ weight loss at 1 month $(b=0.276, P<0.001)$ but not procedure type $(B=0.02, P=0.63)$ predicted high MDT attendance.

\section{Discussion}

We report 1 year weight loss outcome of four EBT techniques in a structured bariatric program and present the predictive variables for better results. We found the Apollo ESG demonstrated higher weight loss results at the end of 1 year. However, in regression analysis, adherence to MDT follow-up predicted weight loss at 1 year independent of procedure type. Only $50 \%$ of patients completed follow-up, and among them, endoscopic gastroplasty (Apollo ESG and POSE) promoted more compliance with follow-up appointments. 


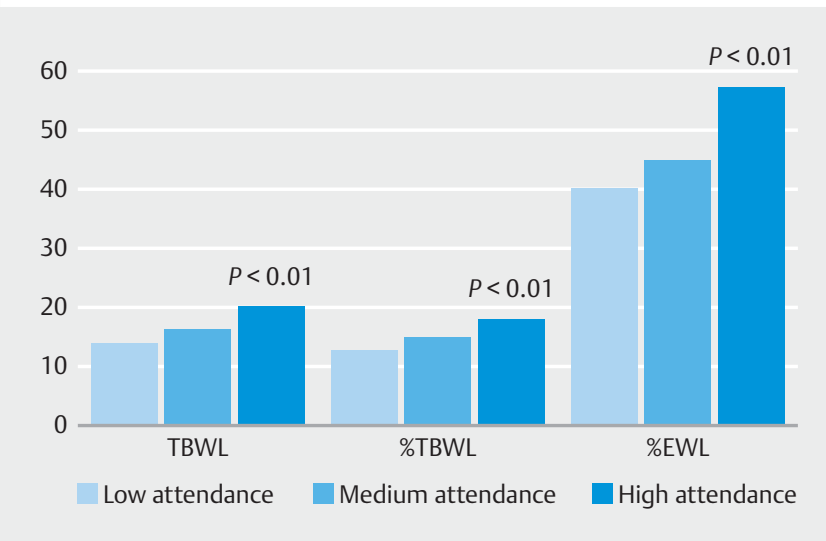

- Fig. 3 Weight loss outcome based on percentage follow-up attendance at 12 months in the completion group $(n=480)$. The best weight loss outcome was observed in the high-attendance group.

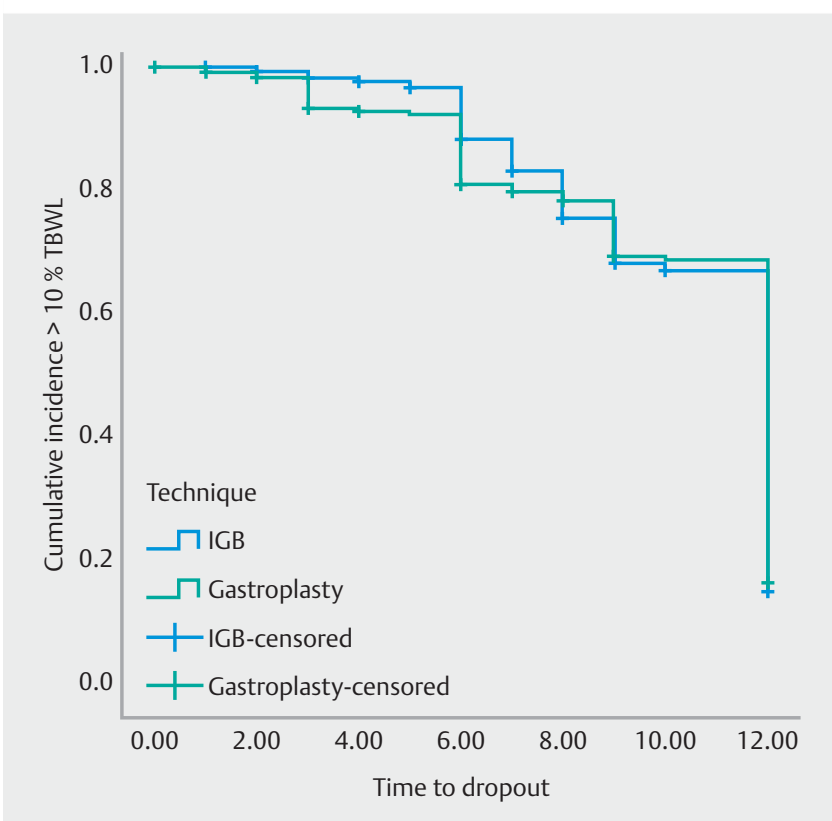

- Fig. 5 Kaplan-Meier curve indicating cumulative incidence of achieving $>10 \%$ TBWL at time of last contact (dropout) with the MDT team in the IGB and EG groups. Procedure type did not influence weight loss results $(0=0.83)$

ESG is a minimally invasive, incisionless endoscopic treatment that functions by shortening and narrowing the gastric volume using a series of full-thickness sutures placed from the distal body to the fundus of the stomach. Weight loss achieved with ESG is related to delay in gastric emptying, reduced hunger, and early satiety [16]. Prior studies have reported that ESG can achieve up to $17 \%$ to $20 \%$ TBWL at 12 and 24 months [7, 22,23]. Weight loss results in our study are consistent with the published literature [24]. There is scant data comparing the outcome of different IGB to EG. A retrospective study $(n=88)$, which is published only in abstract form, showed no significant difference in \%TBWL at 6 months and 1 year between EG and IGB [25]. Our results are in agreement with this finding. We

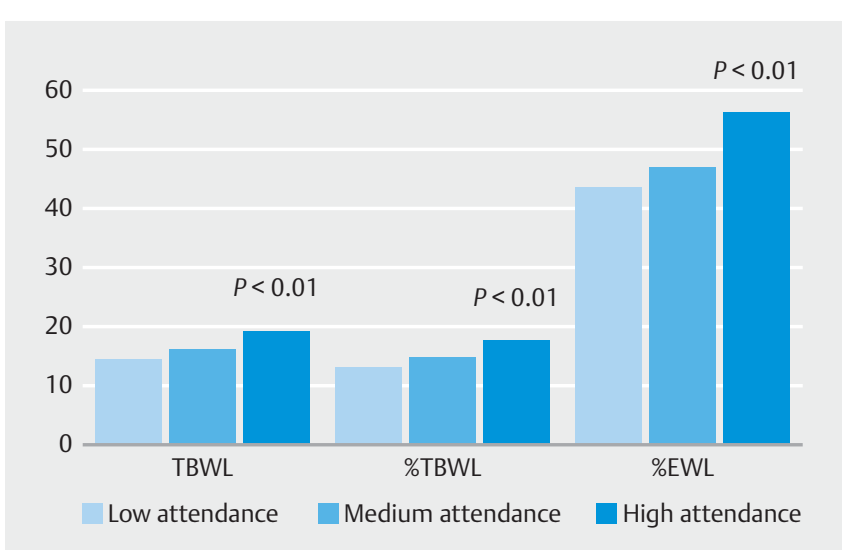

- Fig. 4 Weight loss outcome based on \%follow-up attendance at 6 months in the completion group $(n=624)$. The best weight loss outcome was observed in the high-attendance group.

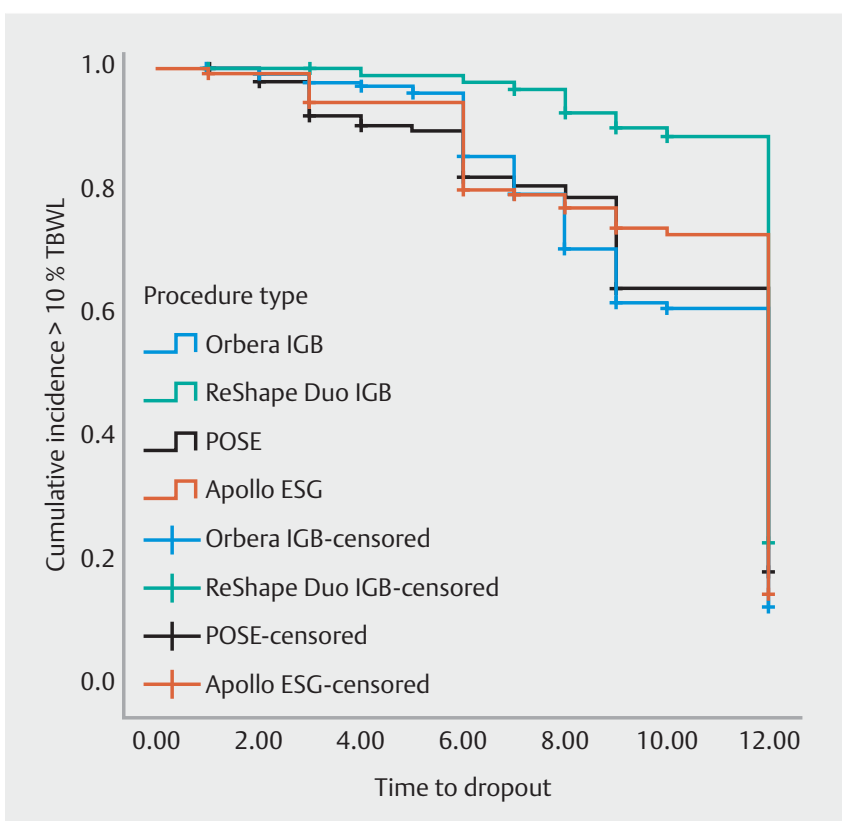

- Fig. 6 Kaplan-Meier curve indicating the cumulative incidence of achieving $>10 \%$ TBWL at time of last contact (dropout) with the MDT team for the four different EBTs.

showed that weight loss at 1 year was not dependant on type of procedure but was determined by follow-up attendance ( $\triangleright$ Fig. 3 and $\triangleright$ Fig. 4). We postulated that frequent interaction with the MDT might have provided an opportunity to identify "at risk of failure" patients and intervene at an early stage. Besides, the psychological counseling and motivation of early responders may have promoted sustained weight loss at 1 year.

Despite its significance, non-adherence and loss to follow-up is still an unresolved problem with many obesity treatments $[26,27]$. We observed that half the patients were lost to follow-up at 1 year. Our drop-out rates were similar to those described in bariatric surgical series and with conventional therapies [28-30]. Surgical studies have identified several variables 
- Table 6 Logistic regression assessing predictive factors for follow-up completion in 962 patients.

\begin{tabular}{|c|c|c|c|c|c|c|}
\hline \multirow[t]{2}{*}{ Covariables } & \multicolumn{2}{|c|}{ 6-months } & \multicolumn{4}{|c|}{1 year } \\
\hline & $\operatorname{Exp}(B)$ & $95 \% \mathrm{Cl}$ & $P$ value & $\operatorname{Exp}(B)$ & $95 \% \mathrm{Cl}$ & $P$ value \\
\hline Gender & 1.17 & $0.77-1.78$ & 0.45 & 1.64 & $1.11-2.42$ & 0.01 \\
\hline Age & 0.99 & $0.9-1.01$ & 0.67 & 1.004 & $0.99-1.02$ & 0.63 \\
\hline Initial BMI & 1.04 & $1.01-1.77$ & 0.01 & 1.82 & $1.25-2.64$ & $<0.001$ \\
\hline Procedure type & 1.28 & $0.86-1.89$ & 0.21 & 1.44 & $1.0-2.07$ & 0.04 \\
\hline 1-month \%weight loss & 1.07 & $1.01-1.14$ & 0.02 & 1.134 & $1.07-1.20$ & $<0.001$ \\
\hline
\end{tabular}

for long-term success but have a limited description of followup compliance. In the current study, we found that EG procedure (Apollo ESG, POSE), female sex, and high initial BMI predicted higher follow-up adherence at 1 year. It is understandable that EG, a more lasting treatment option, promoted more follow-up adherence than the temporary IGBs, which are usually removed at 6 months. However, the finding that high BMI, independent of the patient's age, predicts adherence is in contrast to published studies. In fact, among bariatric surgical cases, high BMI and advanced age are considered as poor predictors for treatment compliance [28, 31, 32]. We are uncertain about the reason for this discrepancy. The cohort of patients with high BMI who choose EBT are those who declined surgery because of its invasiveness and risk. We believe these patients are different from bariatric surgical patients and that they are more motivated to achieve results with a less invasive treatment option. Increased adherence to follow-up observed among patients who achieved significant weight loss at 1 month lends support to this hypothesis. Also, achievement of early desired weight loss in the low BMI group may have given them confidence to self-manage their condition and drop-out from follow-up.

Our study has several strengths. It represents one of the largest bariatric endoscopy cohorts to date that has the weight loss outcome recorded for different EBTs at the end of 1 year. Performance of the procedures by the same endoscopist eliminated technique-related bias. Most centers tend to specialize in one technique and focus on performing only a specific type of EBT. Also, they differ considerably in their nutritional plan and follow-up protocol. Thus, to study and compare the outcome of different EBTs from a heterogenous bariatric endoscopy program may be difficult. We have standardized the follow-up program, built an MDT team with experience in bariatric endoscopy care, and have demonstrated the importance of the MDT program in achieving weight loss at 1 year. We have also added new information on predictive factors for weight loss and follow-up compliance in the EBT cohort, which were previously less described.

Our study has certain limitations which are inherent to a retrospective analysis. The study population was mainly Spanish, and all the procedures were performed by a single endoscopist in a single center. Reproducibility of similar results in other centers needs to be studied. Although our post-procedure followup was standardized across different EBTs, there was a slight variation in nutrition escalation during the first month between IGBs and EG. It possibly could have contributed to excess weight loss with EG at 1 month but is unlikely to have influenced the outcome at 1 year. We are uncertain whether the demonstrated benefit with adherence to MDT follow-up is a reflection of the true success of our follow-up protocol or the motivation status of the patients. Although challenging to differentiate, our prior experience, and results from bariatric surgical series provide support for the importance of standardized follow-up [17,33]. We did not evaluate some technical factors, such as suture pattern, number of sutures placed and patientrelated factors such as educational status, economic status, comorbidities, prior pharmacotherapy, and mental health in predicting weight loss and compliance to follow-up at 1 year. We could not obtain this information from our database. However, available studies on different ESG patterns have reported no significant difference in weight loss. Similarly, the evidence assessing the role of other parameters in the bariatric surgical series has only yielded conflicting results [31,32,34,35]. In our previous study, we did not find any difference between presence of comorbidities and treatment completion [27]. A prospective study assessing the relevance of these variables in EBT patients ought to be performed.

\section{Conclusion}

In summary, all four EBTs are effective in achieving weight loss within a standardized bariatric endoscopy program at 1 year. In particular, endoscopic gastroplasty procedures (Apollo ESG, POSE) promote higher adherence to MDT follow-up than IGB. Careful monitoring is required in order for low-responder-profile patients (male, BMI $<40 \mathrm{~kg} / \mathrm{m}^{2}$, and less 1 -month $\% \mathrm{TBWL}$ ) to achieve good results. Our study provides insight on the importance of MDT follow-up and centers planning to establish a bariatric program should focus on building a robust MDT and not just focus on the procedures alone. 
Competing interests

Dr. Lopez-Nava is a consultant for Apollo Endosurgery, USA and USGI Medical, USA.

References

[1] Sullivan S, Kumar N et al. ASGE Bariatric Endoscopy Task Force. ASGE position statement on endoscopic bariatric therapies in clinical practice. Gastrointest Endosc 2015; 82: 767 - 772

[2] Abu Dayyeh BK, Edmundowicz S, Thompson CC. Clinical Practice Update: expert review on endoscopic bariatric therapies. Gastroenterology 2017; 152: 716-729

[3] Abu Dayyeh BK et al. ASGE Bariatric Endoscopy Task Force. ASGE Technology Committee. Endoscopic bariatric therapies. Gastrointest Endosc 2015; 81: 1073 - 1086

[4] Bazerbachi F, Vargas Valls E], Abu Dayyeh BK. Recent clinical results of endoscopic bariatric therapies as an obesity intervention. Clin Endosc 2017; 50: $42-50$

[5] Kumar N, Bazerbachi F, Rustagi T et al. The influence of the Orbera intragastric balloon filling volumes on weight loss, tolerability, and adverse events: a systematic review and meta-analysis. Obes Surg 2017; $27: 2272-2278$

[6] Saber AA, Shoar S, Almadani MW et al. Efficacy of first-time intragastric balloon in weight loss: a systematic review and meta-analysis of randomized controlled trials. Obes Surg 2017; 27: 277-287

[7] Lopez-Nava G, Sharaiha RZ, Vargas E] et al. Endoscopic sleeve gastroplasty for obesity: a multicenter study of 248 patients with 24 months follow-Up. Obes Surg 2017; 27: 2649-2655

[8] López-Nava G, Bautista-Castaño I, Jimenez A et al. The Primary Obesity Surgery Endolumenal (POSE) procedure: one-year patient weight loss and safety outcomes. Surg Obes Relat Dis 2015; 11: 861 - 865

[9] Kumar N. Weight loss endoscopy: Development, applications, and current status. World J Gastroenterol 2016; 22: 7069-7079

[10] Alqahtani A, Al-Darwish A, Mahmoud AE et al. Short-term outcomes of endoscopic sleeve gastroplasty in 1000 consecutive patients. Gastrointest Endosc 2019; 89: $1132-1138$

[11] Sharaiha RZ, Kumta NA, Saumoy M et al. Endoscopic sleeve gastroplasty significantly reduces body mass index and metabolic complications in obese patients. Clin Gastroenterol Hepatol 2017; 15: $504-$ 510

[12] Abu Dayyeh BK, Kumar N et al. ASGE Bariatric Endoscopy Task Force and ASGE Technology Committee. ASGE Bariatric Endoscopy Task Force systematic review and meta-analysis assessing the ASGE PIVI thresholds for adopting endoscopic bariatric therapies. Gastrointest Endosc 2015; 82: 425-438

[13] Al-Bawardy B, Mukewar SS, Genco A et al. Mo1549 meta-analysis of the orbera intragastric balloon for the endoscopic management of obesity. Gastrointest Endosc 2015; 81: AB462

[14] Bazerbachi F, Haffar S, Sawas T et al. Fluid-filled versus gas-filled intragastric balloons as obesity interventions: a network meta-analysis of randomized trials. Obes Surg 2018; 28: 2617-2625

[15] Agnihotri A, Xie A, Bartalos C et al. Real-world safety and efficacy of fluid-filled dual intragastric balloon for weight loss. Clin Gastroenterol Hepatol 2018; 16: 1081 -1088.e1

[16] Abu Dayyeh BK, Acosta A, Camilleri M et al. Endoscopic sleeve gastroplasty alters gastric physiology and induces loss of body weight in obese individuals. Clin Gastroenterol Hepatol 2017; 15: 37-43
[17] Lopez-Nava G, Galvao M, Bautista-Castaño I et al. Endoscopic sleeve gastroplasty with 1-year follow-up: factors predictive of success. Endosc Int Open 2016; 4: E222 -E227

[18] Lopez-Nava G, Rubio MA, Prados $S$ et al. BioEnterics ${ }^{\circledR}$ intragastric balIoon $\left(\mathrm{BIB}^{\circledR}\right)$. Single ambulatory center Spanish experience with 714 consecutive patients treated with one or two consecutive balloons. Obes Surg 2011; 21: 5-9

[19] Lopez-Nava G, Bautista-Castaño I, Jimenez-Baños A et al. Dual intragastric balloon: single ambulatory center Spanish experience with 60 patients in endoscopic weight loss management. Obes Surg 2015; 25 : $2263-2267$

[20] Khan Z, Khan MA, Hajifathalian K et al. Efficacy of endoscopic interventions for the management of obesity: a meta-analysis to compare endoscopic sleeve gastroplasty, aspireassist, and primary obesity surgery. Endolumenal Obes Surg 2019; 29: 2287-2298

[21] Aranceta Bartrina], Arija ValV et al. Grupo Colaborativo de la Sociedad Española de Nutrición Comunitaria (SENC). Dietary guidelines for the Spanish population (SENC, December 2016); the new graphic icon of healthy nutrition. Nutr Hosp 2016; 33: 1-48

[22] Sharaiha RZ, Kedia P, Kumta N et al. Initial experience with endoscopic sleeve gastroplasty: technical success and reproducibility in the bariatric population. Endoscopy 2015; 47: 164-166

[23] Kumar N, Abu Dayyeh BK, Lopez-Nava BreviereG et al. Endoscopic sutured gastroplasty: procedure evolution from first-in-man cases through current technique. Surg Endosc 2018; 32: 2159-2164

[24] Li P, Ma B, Gong S et al. Efficacy and safety of endoscopic sleeve gastroplasty for obesity patients: a meta-analysis. Surg Endosc 2019: doi:10.1007/s00464-019-06889-6 [Epub ahead of print]

[25] Fayad L, Adam A, Hill C et al. Endoscopic sleeve gastroplasty versus intragastric balloons for the management of obesity. Gastrointest Endosc 2018; 87: AB609-AB610

[26] Sala M, Haller DL, Laferrère $B$ et al. Predictors of attrition before and after bariatric surgery. Obes Surg 2017; 27: 548 - 551

[27] Bautista-Castaño I, Molina-Cabrillana J, Montoya-Alonso JA et al. Variables predictive of adherence to diet and physical activity recommendations in the treatment of obesity and overweight, in a group of Spanish subjects. Int J Obes Relat Metab Disor 2004; 28: 697-705

[28] Wood GC, Benotti PN, Lee C] et al. Evaluation of the association between preoperative clinical factors and long-term weight loss after Roux-en-Y gastric bypass. JAMA Surg 2016; 151: 1056 - 1062

[29] Pontiroli AE, Fossati A, Vedani P et al. Post-surgery adherence to scheduled visits and compliance, more than personality disorders, predict the outcome of bariatric restrictive surgery in morbidly obese patients. Obes Surg 2007; 17: $1492-1497$

[30] Ortega E, Morínigo R, Flores L et al. Predictive factors of excess body weight loss 1 year after laparoscopic bariatric surgery. Surg Endosc 2012; 26: $1744-1750$

[31] Goldenshluger A, Elazary R, Cohen MJ et al. Predictors for adherence to multidisciplinary follow-up care after sleeve gastrectomy. Obes Surg 2018; 28: $3054-3061$

[32] Sillén L, Andersson E. Patient factors predicting weight loss after Roux-en-Y gastric bypass. J Obes 2017; 2017: 3278751

[33] Kaiser KA, Franks SF, Smith AB. Positive relationship between support group attendance and one-year post-operative weight loss in gastric banding patients. Surg Obesity Relat Dis 2011; 7: 89-93

[34] McVay MA, Friedman KE, Applegate KL et al. Patient predictors of follow-up care attendance in Roux-en-Y gastric bypass patients. Surg Obes Relat Dis 2013; 9: $956-962$

[35] Odom J, Zalesin KC, Washington TL et al. Behavioral predictors of weight regain after bariatric surgery. Obes Surg 2010; 20: 349-356 